\title{
Movement of the Trash Inside of Fiber Material When Available Elastic Force of Clutch
}

\author{
Ilkhom Madumarov*, Botirjon Mardonov, Rakhmatjon Ruzmetov, Temur Tuychiev \\ Tashkent Institute of Textile and Light Industry, Tashkent, Uzbekistan \\ Email: *imadumarov61@mail.ru
}

How to cite this paper: Madumarov, I., Mardonov, B., Ruzmetov, R. and Tuychiev, T. (2018) Movement of the Trash Inside of Fiber Material When Available Elastic Force of Clutch. Engineering, 10, 579-587. https://doi.org/10.4236/eng.2018.109042

Received: July 16, 2018

Accepted: August 31, 2018

Published: September 3, 2018

Copyright ( $\odot 2018$ by authors and Scientific Research Publishing Inc. This work is licensed under the Creative Commons Attribution International License (CC BY 4.0).

http://creativecommons.org/licenses/by/4.0/

(c) (i) Open Access

\begin{abstract}
In the article, the motions of the magpies inside the fibrous material are studied in the presence of an elastic bonding force and the force of dry friction. It is assumed that the volatility of raw cotton in the form of a ball hits the initial velocity about the grate, and after the impact the velocity of the center of mass of the volatility is zero. Until the moment of impact, the mote is located along the beam, which tilts to the horizon and has the same velocity with the center of the fly, and after the impact it moves along this ray. The case is considered when the coefficients of dynamic rigidity, adhesion strength and coefficient of friction depend on the temperature difference between the initial and the current, and it is assumed that these parameters decrease with increasing temperature difference. Accordingly, in the equation of motion of the mote, the sign of the speed of movement of the magpies is taken into account in the direction of the force of contact between it and the fibrous mass. Different stages of the motion of the speck along the ray are considered. On the basis of the equations obtained, the trajectories and the geometric locations of the points of location of the magpies located along the ray are established.
\end{abstract}

\section{Keywords}

Cotton, Fiber, Mote, Trash, Temperature, Grate, Cotton Seed, Cotton Cleaning

\section{Introduction}

The productivity of the machine, the humidity, the contamination, the degree of preparedness of the material for cleaning [1], which relate to both raw cotton and cotton fiber, lint and fibrous waste, have a great influence on the efficiency of isolating impurities from the fibrous material. A number of researchers established the dependence of the cleaning effect on the named parameters, and rec- 
ommended the optimal moisture parameters of the material and the productivity of the corresponding machine, depending on the initial contamination and moisture content of the material being processed [2] [3] [4] [5]. However, the mechanism for extracting weedy impurities from the composition of the pulp has not yet been fully disclosed, and factors that affect the coupling of trash with fibrous material have not been established. The proposed recommendations are not sufficiently substantiated (especially theoretically). Theoretically, the effect of temperature on the process of recovering impurities is not studied. The experimental data obtained in [2] [3] [4] show a significant decrease in the magnitude of the contact force between impurities and the pulp. There are still ways to increase the efficiency of the cleaning process from weeds; it is required to establish the optimum temperature and humidity condition of raw cotton.

\section{Main Part}

Let the particle of raw cotton strike with the initial speed $v_{0}$ about the grate. We assume that after impact the velocity of the center of mass (the seed nucleus) is zero. Prior to the impact, the mote with mass $m$ is located along the beam, which forms an angle $\varphi_{0}$ with the horizon. Before the impact, the mote has the same speed with the core and after the impact it moves along the $\mathrm{OM}_{0}$ beam. Then, after the impact, the equation of motion of the speck along the ray is written as:

$$
\begin{gathered}
m \frac{\mathrm{d}^{2} x}{\mathrm{~d} \tau^{2}}=-c_{1} x+m g \sin \varphi_{0}-\left[f_{1} m g \cos \varphi_{0}+q_{1}\right] \cdot \operatorname{sign} \dot{x} \\
\text { sign } \dot{x}= \begin{cases}+1 & x>0 \\
-1 & x<0\end{cases}
\end{gathered}
$$

where: $\tau$-time, $x$-movement of a speck along the ray $\mathrm{OM}_{0}, c_{1}$-dynamic stiffness; $q_{1}$-coupling force; $m$-mass of trash, $f_{1}$-coefficient of friction, between the trash and fiber.

Consider the case where the coefficient of dynamic stiffness $c_{1}$, the adhesion force $q_{1}$ and the coefficient of friction $f_{1}$ depend on the temperature difference. According to [6], with increasing temperature these parameters decrease, so we can use such dependences

$$
c_{1}=c_{0} \mathrm{e}^{-\alpha_{c}\left(T-T_{0}\right)}, \quad q_{1}=q_{0} \mathrm{e}^{-\alpha_{q}\left(T-T_{0}\right)}, \quad f_{1}=f_{0} \mathrm{e}^{-\alpha_{f}\left(T-T_{0}\right)}
$$

Here: $c_{0}=c\left(T_{0}\right), q_{0}=q\left(T_{0}\right), f=f_{1}\left(T_{0}\right) \alpha_{c}, \alpha_{q}, \alpha_{f}$-are the coefficients characterizing the rate of decline of the coefficients, determined experimentally; $T_{0}$-is the initial temperature; $T=T(\tau)$-is the current temperature, dependent on time.

The action of the elastic force and the frictional force of the contact to determine the relative displacement of the trash along the fiber is shown in Figure 1.

Initial conditions

$$
x=0, \dot{x}=v_{0} \sin \varphi_{0}>0 \text { at } \tau=0
$$

Equation (2) is written in dimensionless form 


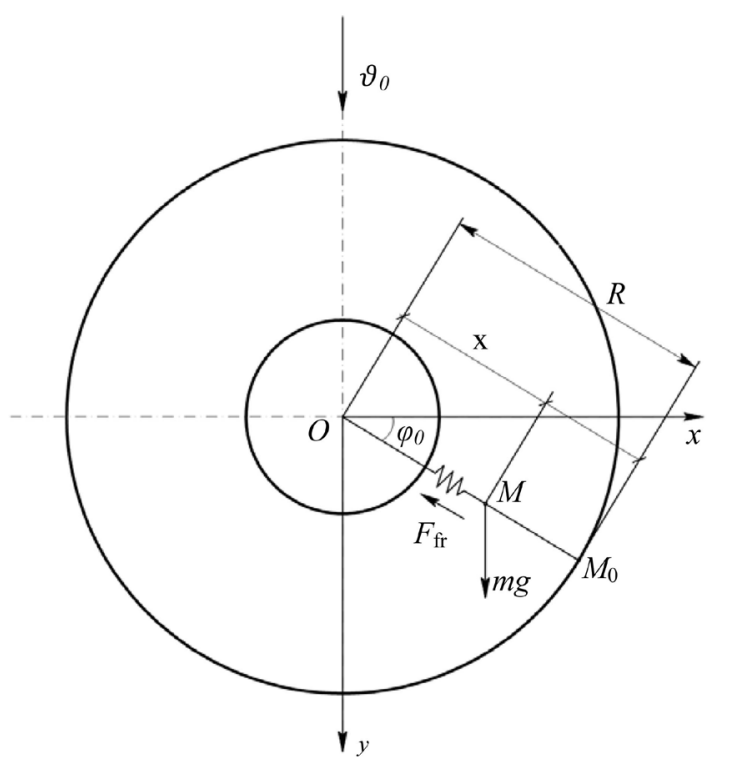

Figure 1. The action of the elastic force and the frictional force of the contact to determine the relative displacement of the trash along the fiber.

$$
\frac{\mathrm{d}^{2} \zeta}{\mathrm{d} \tau_{1}^{2}}=-\zeta \mathrm{e}^{-\alpha_{T}\left(T-T_{0}\right)}+\sin \varphi_{0}-\left[f_{0} \mathrm{e}^{-\alpha_{f}\left(T-T_{0}\right)} \cdot \cos \varphi_{0}+\bar{q} \mathrm{e}^{-\alpha_{q}\left(T-T_{0}\right)}\right] \operatorname{sign} \dot{\zeta}
$$

where: $\zeta=\frac{c_{0}}{m g} x, \tau_{1}=\tau \sqrt{\frac{c_{0}}{m}}, \bar{q}=\frac{q_{0}}{m g}$.

Equation (4) for a given temperature variation with respect to time and coordinate $\zeta$ is integrated numerically.

If we assume that $T=T_{0}$, (in the absence of temperature difference), then the coefficient of Equation (4) does not depend on time and its solution in a dimensionless form is written down

$$
\zeta=\left[\sin \varphi_{0}-f \cos \varphi_{0}-\bar{q}\right]\left(1-\cos \tau_{1}\right)+\bar{V}_{0} \sin \varphi_{0} \sin \tau_{1}
$$

where: $\bar{V}_{0}=\frac{v_{0}}{g} \sqrt{\frac{c_{0}}{m}}$.

Expression (5) describes the law of motion of a speck between fibrous spaces after impact of volatiles on the grate. In the presence of a frictional force on the part of the fibrous mass, the mote at a certain instant of time stops. Its further movement depends on the initial impact velocity.

In the process of motion, part of the initial kinetic energy $\frac{m v_{0}^{2}}{2}$ of the mote passes into the potential energy of compression of the pulp, while the other part proceeds to dissipation due to friction [7] [8].

If the initial velocity is insufficient, then it is possible that all the acquired potential energy of the pulp goes only to dissipation and, therefore, no further movement of the mote is possible, that is, the process does not purify the impact [9] [10]. At some speeds, the accumulated potential energy of the pulp will be sufficient to inform the mote of the motion in the opposite direction. In this 
case, the next stage of the movement begins, in which the mote makes movement with respect to the center of the volatility according to the vibrational law. Further movement towards the center of the flying, as noted above, again depends on the initial speed.

From (6) we find the speed of the mote

$$
\dot{\zeta}=\left[\sin \varphi_{0}-f \cos \varphi_{0}-\bar{q}\right] \sin \tau_{1}+\bar{v}_{0} \sin \varphi_{0} \cos \tau_{1}
$$

At some point in time, the mote stops, that is

$$
\dot{\zeta}=\left[\sin \varphi_{0}-f \cos \varphi_{0}-\bar{q}\right] \sin \tau_{0}+\bar{v}_{0} \sin \varphi_{0} \cos \tau_{0}=0
$$

From which we get:

$$
\tau_{0}=\pi-\alpha
$$

where: $\alpha=\operatorname{arctg} \frac{\bar{v}_{0} \sin \varphi_{0}}{a}$.

When $\tau>\tau_{0}$ moving in the opposite direction is possible with the condition if $\operatorname{sign} \dot{\xi}=-1, \dot{\xi} \leq 0$ at $\tau>\tau_{0}$ which gives

$$
-\xi_{10}+\sin \varphi_{0}+f \cos \varphi_{0}+\bar{q} \leq 0
$$

where: $\xi_{10}=\xi\left(\tau_{0}\right)=a\left(1-\cos \tau_{0}\right)+v_{0} \sin \varphi_{0} \cdot \sin \tau_{0}, a=\sin \varphi_{0}-b$, $b=f \cos \varphi_{0}+\bar{q}$.

Inequalities (6) give

$$
\left(\sin \varphi_{0}-f \cos \varphi_{0}+\bar{q}\right)\left(1-\cos \tau_{0}\right)-\bar{v}_{0} \sin \varphi_{0} \sin \tau_{0}+\sin \varphi_{0}+f \cos \varphi_{0}+\bar{q} \leq 0
$$

or

$$
-a \cos \tau_{0}+\bar{v}_{0} \sin \varphi_{0} \cdot \sin \tau_{0} \geq 2 b
$$

Hence we obtain the condition for the velocity

$$
\sqrt{a^{2}+v_{0}^{2} \cdot \sin ^{2} \varphi_{0}} \geq 2 b
$$

If condition (7) is not satisfied, then the mote is further in a state of rest. When condition (7) is satisfied, the motion of the mote is described by equation

$$
\ddot{\xi}^{\prime}=-\xi_{10}-a_{1}
$$

where: $a_{1}=\sin \varphi_{0}+f \cos \varphi_{0}+\bar{q}=\sin \varphi_{0}+b$.

The solution of this equation with the initial conditions

$$
\xi=\xi_{10}, \quad \xi=0
$$

has the form:

$$
\xi=\left(\xi_{10}-a_{1}\right) \cos \left(\tau-\tau_{0}\right)+a_{1}
$$

This stage of the movement continues until the time $\dot{\xi}=0$ at which, whence we have

$$
\tau=\tau^{(1)}=2 \pi-\alpha
$$

The next stage of the movement (that is, movement of the mote from the center) is possible, if

$$
\xi_{20}+a \geq 0
$$


where: $\xi_{20}=\xi\left(\tau_{1}\right)$ or $\xi_{10}-2 a_{1}+a \geq 0$.

We get

$$
\xi_{10} \geq 2 a_{1}-a=\sin \varphi_{0}+3 f \cos \varphi_{0}+\bar{q}=\sin \varphi_{0}+3 b
$$

substituting the value $\xi_{10}$, we obtain condition

$$
\sqrt{a^{2}+\bar{v}_{0}^{2} \cdot \sin ^{2} \varphi_{0}} \geq 4 b
$$

The remaining stages of the motion of the mote are determined in a similar way, for example, before the $n$th stage of the motion is possible, if

$$
\sqrt{a^{2}+\bar{v}_{0}^{2} \cdot \sin ^{2} \varphi_{0}} \geq 2 n b \quad(n=1,2,3, \cdots)
$$

If $a<0$ is $b>\sin \varphi_{0}$, then the time at which the speed of the mote turns to zero is determined by the formula

$$
\tau_{0}=\operatorname{arctg} \frac{v_{0} \sin \varphi_{0}}{b-\sin \varphi_{0}}
$$

the remaining formulas remain unchanged.

With the help of the obtained dependences, it is possible to establish a condition when performing a certain mote does not penetrate deep into the pulp. Let us consider the case $a<0$. Let the mote at $t=0$ be at a distance $\xi_{0}$ from the surface of the seed. Then, after the impact, it moves away from this position by a distance

$$
\xi_{\max }=\xi_{10}=a+\sqrt{a^{2}+\sin ^{2} \varphi_{0}}=\sin \varphi_{0}-b+\sqrt{\left(\sin \varphi_{0}-b\right)^{2}+v_{0}^{2} \sin ^{2} \varphi_{0}}
$$

if the conditions

$$
\sqrt{a^{2}+\bar{v}_{0}^{2} \cdot \sin ^{2} \varphi_{0}} \geq 2 b, \quad \xi_{0}+\xi_{\max } \leq \bar{R}, \quad \bar{R}=\frac{R_{c}}{m g}
$$

where: $R_{C}$-is the radius of the fibrous mass ball.

Then the mote makes a reverse movement to the center and the depth of maximum penetration will be equal to

$$
\xi_{20}=2 a_{1}-\xi_{10}=\sin \varphi_{0}+3 b-\sqrt{a^{2}+\bar{v}_{0}^{2}} \cdot \sin ^{2} \varphi_{0}
$$

If $\xi_{20}>0$, then the mote becomes deeper into the mass before reaching its original position $\xi_{10}$. If $\xi_{20}<0$ it will be introduced into the depth of the ball. In the first case we have:

$$
2 b \leq \sqrt{a^{2}+\bar{v}_{0}^{2}} \sin ^{2} \varphi_{0} \leq 3 b+\sin \varphi_{0}
$$

in the second case

$$
\sqrt{a^{2}+\bar{v}_{0}^{2} \cdot \sin ^{2} \varphi_{0}} \geq 3 b+\sin \varphi_{0}
$$

Now let us $a>0$ set

$$
a=+\lambda^{2}=\sin \varphi_{0}-b, \quad\left(b<\sin \varphi_{0}\right)
$$

then

$$
\xi_{10}=\sqrt{\lambda^{4}+v_{0}^{2} \cdot \sin ^{2} \varphi_{0}}-\lambda^{2}=\sqrt{\left(b-\sin \varphi_{0}\right)^{2}+v_{0}^{2} \sin \varphi_{0}}+b-\sin \varphi_{0}
$$




$$
\begin{aligned}
\xi_{20} & =2 a_{1}-\xi_{10}=2\left(\sin \varphi_{0}+b\right)-\sqrt{\left(b-\sin \varphi_{0}\right)^{2}+\bar{v}_{0}^{2} \cdot \sin ^{2} \varphi_{0}}-b+\sin \varphi_{0} \\
& =2 \sin \varphi_{0}+2 b-\sqrt{\left(b-\sin \varphi_{0}\right)^{2}+\bar{v}_{0}^{2} \sin ^{2} \varphi_{0}}-b-\sin \varphi_{0} \\
& =3 \sin \varphi_{0}+b-\sqrt{\left(b-\sin \varphi_{0}\right)^{2}+\bar{v}_{0}^{2} \cdot \sin ^{2} \varphi_{0}}
\end{aligned}
$$

Thus, if

$$
2 b \leq \sqrt{\left(b^{2}-\sin \varphi_{0}\right)^{2}+\bar{v}_{0}^{2} \sin ^{2} \varphi_{0}} \leq b+3 \sin \varphi_{0}
$$

then the mote does not reach its original position. If

$$
\sqrt{\left(b^{2}-\sin \varphi_{0}\right)^{2}+\bar{v}_{0}^{2} \sin ^{2} \varphi_{0}} \geq b+3 \sin \varphi_{0}
$$

then there are additional depressions of the mote into the fibrous mass.

To assess the effect of changes in the stiffness coefficients $c_{1}, f_{1}$ and the adhesion force $q_{1}$ on temperature, experimental studies were carried out [6].

For constants $f_{1}$ and $q_{1}$ with a change in the stiffness coefficient $c_{1}$ according to Formula (1), we should write Equation (4) in the form:

$$
\frac{\mathrm{d}^{2} \zeta}{\mathrm{d} \tau^{2}}=\zeta \mathrm{e}^{-\alpha_{c}\left(T-T_{0}\right)}+\sin \varphi_{0}-\left(f \cos \varphi_{0}+\bar{q}\right) \operatorname{sign} \dot{\zeta}
$$

and the solution of Equation (8) at $T=T_{1}=$ const can be represented in the form:

$$
\zeta=\left(\sin \varphi_{0}-f \cos \varphi_{0}-\bar{q}\right)\left(1-\cos \zeta_{1} \tau_{1}\right)+\dot{v}_{0} \sin \varphi_{0} \sin \zeta_{1} \tau_{1}
$$

where: $\zeta_{1}=\mathrm{e}^{-\alpha_{c}\left(T_{1}-T_{0}\right)}$.

\section{Analysis of Results}

As can be seen, the solutions of the equation of motion of the mote, in each stage are presented in an analytical form, which includes mechanical parameters. Comparing the types of solutions (9) with (5) that correspond to the 1 and 2 stages of the movement, we note that the change in the stiffness factor affects the stop and mobility time values at each stage, the movement of weed impurities. For example, the stopping time of the mote changes by an amount $\frac{\tau_{0}}{\zeta_{1}}$ because $\zeta_{1}<1$, then the stopping time increases. For a constant value of the stiffness coefficient $c=c_{0} \cdot \mathrm{e}^{-\alpha_{c}\left(T-T_{0}\right)}$, the parameters $f_{1}$ and $q_{1}$ in a range of temperature variations were represented by the dependences (1) where $\alpha_{c}=0.11 / \mathrm{deg}$ is assumed, $T=T_{1}=$ const, calculations show that the temperature change significantly affects the parameters $\tau_{0}$ and the mixing of the mote.

Thus, the obtained data show that as the temperature of the fibrous cover increases, the intensity of excretion of impurities from cotton increases.

On the basis of the equations obtained, it is possible at an arbitrary time to establish the trajectory and the locus of the points of finding the magpies along the rays $\mathrm{OM}_{0}$. These trajectories make it possible to determine the number of isolated trashes, depending on the depth of their lying in the fibrous mass. 
At small angles $\varphi_{0}$, the mote at low temperatures (Figure 2, Curves 1,2) makes one oscillation toward the positive direction. As the temperature rises, the mote can move in the opposite direction, and due to the decrease in the resistance force it can make two oscillations (Curve 5). In this case, the amplitudes have the greatest value in positive directions, and the effect of the temperature drop on the values of these amplitudes is insignificant. The results presented in Figure 3 and Figure 4 show that the orientation of the speck with respect to the horizon can largely affect the character of the movement of the mote in the composition of the pulp. In this case, active movement of the mote is detected in both positive and negative directions. In this case, the mote can make several oscillations along the beam. The maximum value of the amplitude of the oscillations in the positive direction is practically independent of the temperature drop, while the penetration of the mote into the interior of the fibrous mass occurs with smaller amplitudes. Thus, when cleaning the pulp from the mote, it is rational to choose the direction of impact and the value of the temperature drop.

Figures 2-4 show a graph of the change in the movement of the mote located in a fibrous medium, in the case of inequality

$$
a>0 \text { and } 6 b \leq \sqrt{a^{2}+\bar{v}_{0}^{2} \cdot \sin ^{2} \varphi_{0}} \leq 8 b
$$

at different temperatures $\left(1 . T=20^{\circ} \mathrm{C} ; 2 . T=30^{\circ} \mathrm{C} ; 3 . T=40^{\circ} \mathrm{C} ; 4 . T=50^{\circ} \mathrm{C} ; 5 . T\right.$ $=60^{\circ} \mathrm{C}$ ) and angle $\varphi_{0}$.

It is shown that as the temperature of the fiber increases, the amplitude of displacement of the litter increases. For example, at a fiber temperature $t_{B}=20^{\circ} \mathrm{C}$, the amplitude of the trash displacement ends in the second stage, and at a fiber temperature $t_{B}=50^{\circ} \mathrm{C}$ on the third.

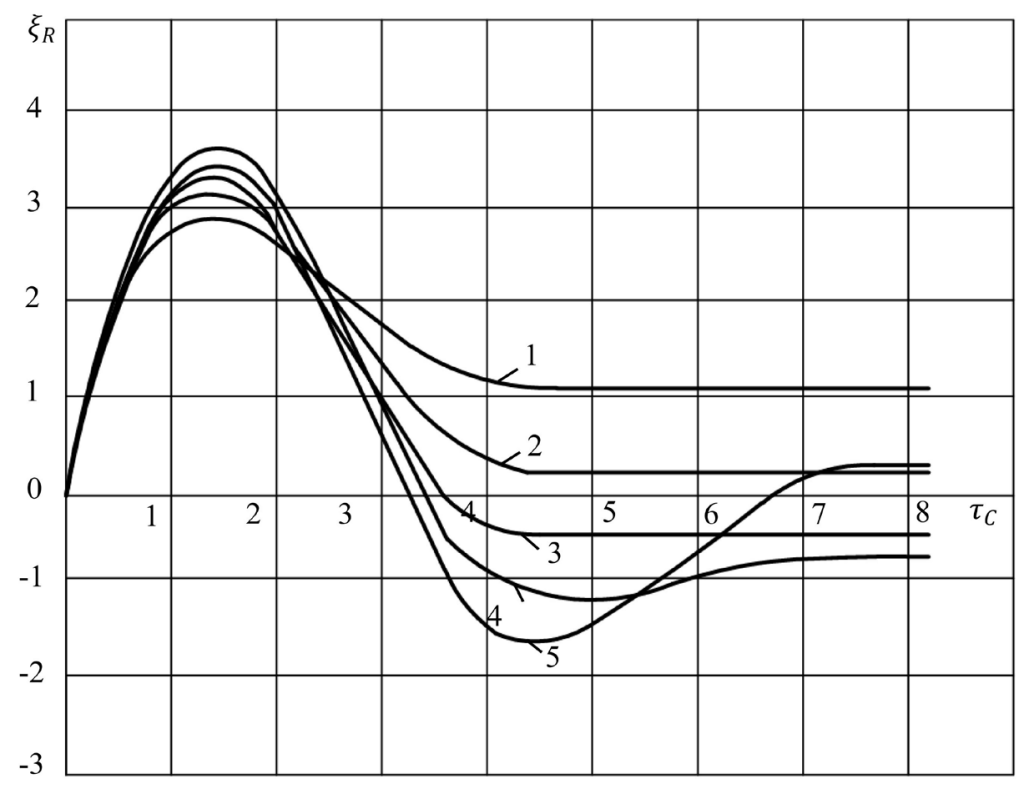

$1,2,3,4,5$, respectively, $\mathbf{t}_{\mathrm{B}}=20^{\circ} \mathrm{C} ; 30^{\circ} \mathrm{C} ; 40^{\circ} \mathrm{C} ; 50^{\circ} \mathrm{C} ; 60^{\circ} \mathrm{C}$

Figure 2. The movement of litter within the pulp at various temperatures and angles $\varphi_{0}=15^{\circ}$. 


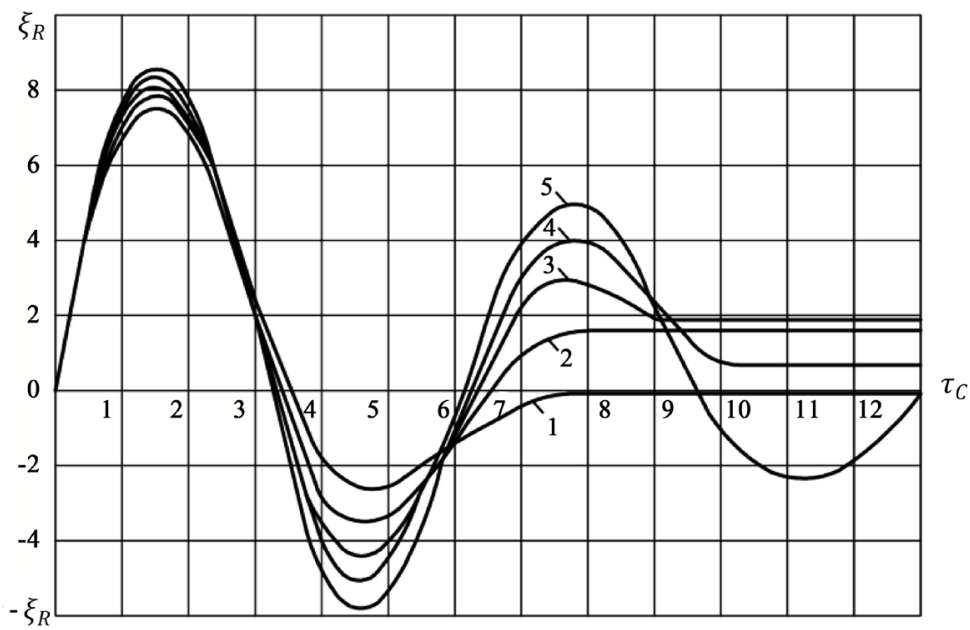

$1,2,3,4,5$, respectively, $\mathrm{t}_{\mathrm{B}}=20^{\circ} \mathrm{C} ; 30^{\circ} \mathrm{C} ; 40^{\circ} \mathrm{C} ; 50^{\circ} \mathrm{C} ; 60^{\circ} \mathrm{C}$

Figure 3. The movement of litter within the pulp at various temperatures and angles $\varphi_{0}=30^{\circ}$.

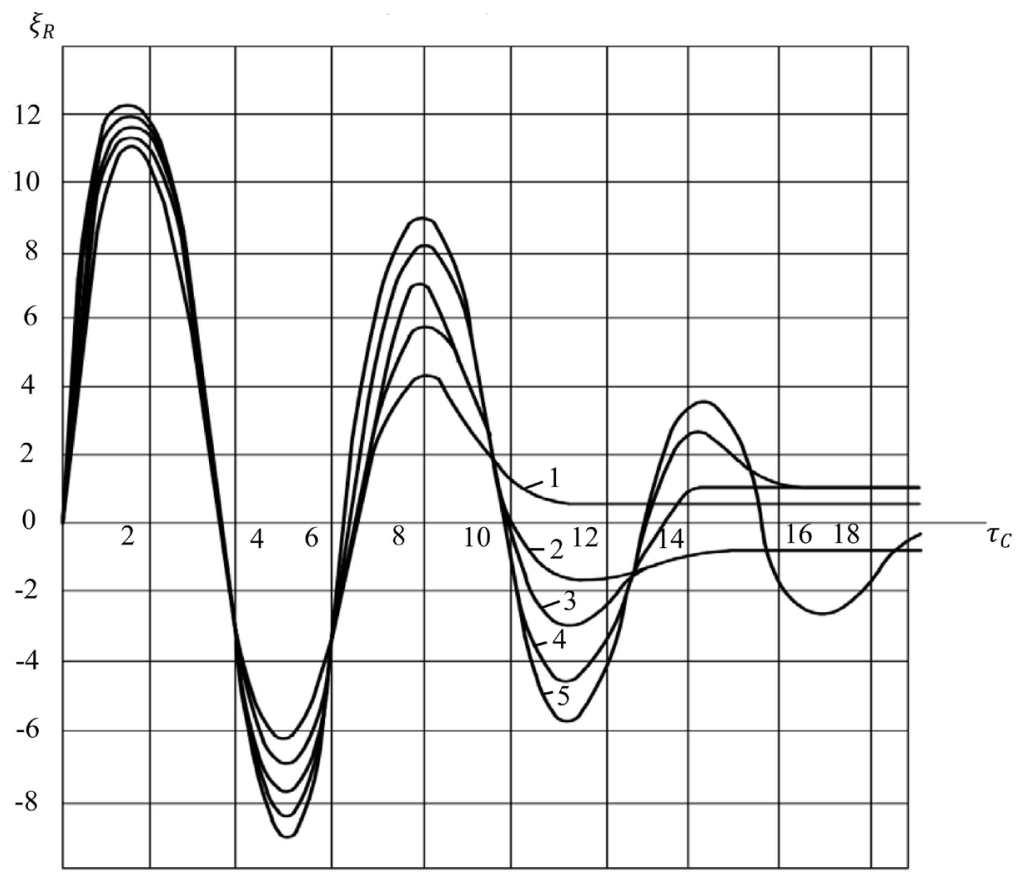

$1,2,3,4,5$, respectively, $\mathrm{t}_{\mathrm{B}}=20^{\circ} \mathrm{C} ; 30^{\circ} \mathrm{C} ; 40^{\circ} \mathrm{C} ; 50^{\circ} \mathrm{C} ; 60^{\circ} \mathrm{C}$

Figure 4. The movement of litter within the pulp at various temperatures and angles $\varphi_{0}=45^{\circ}$.

\section{Conclusions}

Thus, under impact, a part of the garbage that is on the surface of the cotton particle is removed, and some of it moves into the inside of the particle. The depth of garbage deposition on impact increases or decreases depending on the location of their relatively hitting surfaces and the temperature of the fiber.

Theoretical studies of the process of cleaning raw cotton under shock effects, taking into account the effect of temperature, show that as the temperature of 
the fiber increases, the amplitude of movement of the mote increases, located in the fibrous medium, and intense sorption of the pulp from the pulp takes place.

\section{Conflicts of Interest}

The authors declare no conflicts of interest regarding the publication of this paper.

\section{References}

[1] Tuychiev, T.O. (2018) Increasing to Efficiency of the Cleaning Machine to Improvements Feeders and Preparing the Raw Cotton to Technological Process to Cleaning. PhD Dissertation, Tashkent, 120.

[2] Parpiev, A.P., Mardonov, B.M., Madumarov, I.D. and Tuychiev, T.O. (2013) Simulation of Mass Movement of Raw Cotton in the Feeder Zone. Problems of Textiles, No. 1, 81-86.

[3] Madumarov, I.D., Mardonov, B.M. and Tuychiev, T.O. (2017) Investigation of the Movement of Raw Cotton in the Zone of Its Interaction with the Mesh Surface. Problems of Textiles, No. 3, 21-26.

[4] Korabelnikov, A., Rudovsky, P., Sarimsakov, O. and Gaibnazarov, E. (2018) Dynamics of the Movement of Weed Particles in the Pulp during Its Purification in Order to Establish the Rational Parameters of the Process. Materials of the International Scientific Conference, NamIIT, Namangan, 125.

[5] Burnashev, R.Z., Muradov, R. and Sarimsakov, O. (2001) Dynamic Task of Interaction of Raw Cotton with Working Bodies of Processing Machines. Journal of Problems of Mechanics, No. 3-4, 55.

[6] Madumarov, I.D. (1993) Intensification of the Cleaning Process with Optimization of the Heat and Humidity of Raw Cotton. PhD Dissertation, Tashkent, 165.

[7] Madumarov, I.D., Mardonov, B.M. and Tuychiev, T.O. (2017) An Analysis of the Disappearance of Trashes from Fibrous Mass with Its Spatial Motion. Problems of Textiles, No. 4, 35-39.

[8] Tuychiev, T.O., Madumarov, I.D. and Mardonov, B.M. (2017) Investigation of the Process of Release of Dirt Impurities in the Zone of Interaction of It with a Netlike Surface. European Science Review, No. 9-10, 130-133.

[9] Lugachev, A.E. (1998) Development of Theoretical Bases of Feeding and Cotton Cleaning with Reference to the Flow Technology of Its Processing. PhD Dissertation, Tashkent.

[10] Parpiev, A.P. (1988) The Main Integrated Solution of the Problems of Preserving the Fiber Quality and Increasing the Productivity in the Preliminary Processing of Raw Cotton. PhD Dissertation, Kostroma, 438. 